\title{
Performance of Gabor mean Feature Extraction Techniques for Ear Biometrics Recognition System
}

\author{
Bhanu Vadhwani \\ Rajasthan College of Engg. \\ for Women, India
}

\author{
Vineet Khanna \\ JaipuRajasthan College of \\ Engg. for Women
}

\author{
Sandeep Kumar Gupta \\ Machine Learning Research \\ Lab, Jaipur, India
}

\author{
Shubhlakshmi Agarwal \\ The ICFAI University, Jaipur, India
}

\begin{abstract}
Ear biometric recognition is used in a lot of applications as person identification in criminal cases, investigation, and security purpose. Feature optimization stage has an important role for accuracy of correct recognition. Gabor filter have a problem of high dimension and high redundancy. Sampling filter is a problem of not reducing features optimum way. In the proposed Gabor feature extraction technique the Gabor features are filtered using proposed mean filter and obtained optimum features for ear biometric dataset.
\end{abstract}

\section{Keywords}

Ear Biometric Recognition, Gabor Filter, Analysis.

\section{INTRODUCTION}

Human ear biometric is a very useful and powerful source of communicative information about human identification. Auto ear biometric Recognition is used for identification of persons in crime diagnosis, person identification. Feature Selection techniques is used for reduction of redundancy and non pattern part in ear image [1]. Fajri Kurniawan et. al. [2] proposed that

Security system improves by the Biometric systems implemented on several facilities. Gonzalez, Alvarez and Mazorra [3] suggested that feature extraction is a key for ear biometric detection and feature are based on ear curves. Gabor Filter is based on spatial locality, scale, frequency and orientation on facial images [4]. Geometric or Local featurebased approach, Non-Geometric or Appearance or holistic feature based approach and Hybrid approach. Fajri Kurniawan [4] proposed statistical based feature extraction technique using variance for solving occlusion problem of transform based feature extraction technique. Karuna et al. [5] proposed 2 level Gabor filter feature extraction model of ear biometrics recognition while priya et al. [6] modified the karuna's approach using det transformation of facial gesture recognition. Neha et al. analyzed detail survey of Gabor filter [7], dwt[8], det[9] and other feature extraction technique in [10]. In this paper we are proposing 1 level average feature extraction techniques for ear biometrics recognition instead of two level feature extraction for reducing complexity with achieving good accuracy for ear biometrics recognition.

\section{RELATED WORK}

\subsection{Gabor Filter Feature Extraction Technique}

The equation of Gabor filter is given as follows [11]:

$$
\Psi(x, y, \lambda, \theta)=\frac{1}{2 \pi s_{x} s_{y}} e^{-\frac{1}{2}\left(\frac{x_{1}{ }^{2}}{s_{x}^{2}}+\frac{y_{1}{ }^{2}}{s_{y}^{2}}\right)} e^{j \frac{2 \pi x_{1}}{\lambda}}
$$

here, $(\mathrm{x}, \mathrm{y})$, the pixel position in the spatial domain.

$\lambda=1 / \mathrm{f}, \mathrm{f}=$ frequency of pixels.

$\theta,=$ projection angle of Gabor equation.

$\mathrm{S}_{\mathrm{x}}, \mathrm{S}_{\mathrm{y}}$, Standard deviation in $\mathrm{x} \& \mathrm{y}$ directions.

$$
\text { and } \quad \begin{aligned}
\mathrm{x} 1 & =\mathrm{x} \cos \theta+\mathrm{y} \sin \theta \\
\mathrm{y} 1 & =-\mathrm{x} \sin \theta+\mathrm{y} \cos \theta
\end{aligned}
$$

The Gabor features are obtained through convolution of input digital image with Gabor filter bank [12]. $\mathrm{I}(\mathrm{x}, \mathrm{y})$ is a grey scale ear biometric digital image that is of size $\mathrm{M} * \mathrm{~N}$ pixels.

$\mathrm{G}_{\mathrm{u}, \mathrm{v}}(\mathrm{x}, \mathrm{y})=\mathrm{I}(\mathrm{x}, \mathrm{y}) * \Psi(\mathrm{x}, \mathrm{y})$

The convolution operation is performed separately for real and imaginary part[13] of gabor coefficient as equation $5.1 \&$ 5.2.

$$
\begin{aligned}
& \operatorname{Re}(\mathrm{O}(\mathrm{x}, \mathrm{y}))_{\mathrm{m}, \mathrm{n}}=\mathrm{I}(\mathrm{x}, \mathrm{y})^{*} \operatorname{Re}\left(\psi\left(\mathrm{x}, \mathrm{y}, \lambda_{\mathrm{m}}, \theta_{\mathrm{n}}\right)\right) \\
& \operatorname{Im}(\mathrm{O}(\mathrm{x}, \mathrm{y}))_{\mathrm{m}, \mathrm{n}}=\mathrm{I}(\mathrm{x}, \mathrm{y})^{*} \operatorname{Im}\left(\psi\left(\mathrm{x}, \mathrm{y}, \lambda_{\mathrm{m}}, \theta_{\mathrm{n}}\right)\right) \\
& |\mathrm{O}(\mathrm{x}, \mathrm{y})|_{\mathrm{m}, \mathrm{n}}=\left(\left(\operatorname{Re}(\mathrm{O}(\mathrm{x}, \mathrm{y}))_{\mathrm{m}, \mathrm{n}}\right)^{2}+\left(\operatorname{Im}(\mathrm{O}(\mathrm{x}, \mathrm{y}))_{\mathrm{m}, \mathrm{n}}\right)^{2}\right)^{1 / 2}
\end{aligned}
$$

The Gabor feature matrices are multidimensional and have high redundant features so redundancy and dimensions is reduced using filter. The filtered features are kept in feature vector which is passed to classifier for classification. Classification is a supervised leaning process of data mining which is used to determine the class of test data after training [14].

\section{PROPOSED WORK}

To overcome loss of large feature vector dimension, decrease the size of feature vector by executing down sampling without losing any information. For this purpose, the proposed feature selection methods calculate consolidate value of Gabor feature matrices along orientation for each scale for reducing features and redundancy without losing feature values. In the proposed system, 3 scale and 9 different orientations or total $3 * 9=27$, Gabor matrices are generated. Thus process applied a feature reduction of Gabor feature matrices by 9 without losing any information using proposed Mean calculation of different Gabor matrices. This gabor mean matrix is converted into one dimension vector which is called G_mean feature vector and passed to second phase optimization. Second pass feature extraction technique is sampling filtering. 
After sampling, this is final optimized feature vector which is passed to classifier.

\section{EXPERIMENTS \& RESULTS}

Ear biometric recognition system is implemented using Gabor Sampling filter and proposed Gabor average feature extraction. Experimental setup mentioned in Table 1 and performance is evaluated in term of correct recognition rate which is mentioned in Table 2.

Table 1. Experimental setup for implementation of ear biometric recognition System.

\begin{tabular}{|l|l|l|}
\hline Sno. & Parameters & Value \\
\hline 1. & Dataset & AMI Ear biometric Dataset \\
\hline 2. & Preprocessing & $\begin{array}{l}\text { RGB to Gray Scale } \\
\text { Conversion }\end{array}$ \\
\hline 3. & $\begin{array}{l}\text { Feature } \\
\text { Extraction } \\
\text { Technique }\end{array}$ & $\begin{array}{l}\text { Proposed Average Gabor } \\
\text { Scale feature extraction } \\
\text { Technique }\end{array}$ \\
\hline 3. & Classifier & AdaBoost \\
\hline 4. & $\begin{array}{l}\text { Training/Testin } \\
\text { g Ratio }\end{array}$ & 70/30 \\
\hline
\end{tabular}

Table 2. Analysis of recognition rate for technique

\begin{tabular}{|l|l|l|}
\hline Sno & $\begin{array}{l}\text { Feature Extraction } \\
\text { Method }\end{array}$ & $\begin{array}{l}\text { Average } \\
\text { Recognition Rate } \\
(\%)\end{array}$ \\
\hline 1 & $\begin{array}{l}\text { Gabor Sampling Filter } \\
\text { method }\end{array}$ & 65.00 \\
\hline 2 & $\begin{array}{l}\text { Gabor PCA } \\
\text { Extraction Method }\end{array}$ & 77.00 \\
\hline 3 & $\begin{array}{l}\text { Gabor Average DWT } \\
\text { Method [5] }\end{array}$ & 88.5 \\
\hline 4 & $\begin{array}{l}\text { Ear Biometrics using } \\
\text { Proposed Feature } \\
\text { Extraction Techniques }\end{array}$ & 83.5 \\
\hline
\end{tabular}

\section{CONCLUSION}

The results shows that the ear biometric recognition system using Gabor sampling filter have $65 \%$ recognition rate while proposed Gabor filter have $83 \%$ recognition rate. So the performance of proposed Gabor Filter is better than compared to Gabor sampling feature extraction technique for ear biometric recognition system and proposed average Gabor filtering provide better and unique shape pattern about ear classes compared to Gabor sampling Filter.

\section{REFERENCES}

[1] Anshul Khairwal,Kumar Abhishek,Surya Prakash,Tej, “A Comprehensive Study of Various Biometric Identification Techniques", in International Conference on ICCCNT, IEEE, 2012.

[2] Fajri Kurniawan, Mohd. Shafry Mohd. Rahim, "A Review on 2D Ear Recognition", in 8th International Colloquium on Signal Processing and its Applications, IEEE, 2012.
[3] Gonzalez, Alvarez and Mazorra,"Normalization and Feature Extraction on Ear Images", in International Conference on Security Technology, IEEE, 2012.

[4] Bao-Qing Zhang, Zhi-Chun Mul, Hui Zeng, Hong-Bo Huang, "Ear Recogniton Based On Gabor Scale Information", Proceedings of the 2013 International Conference on Wavelet Analysis and Pattern Recognition, Tianjin, 14-17 July, 2013.

[5] Karuna Soni, Umesh Kumar, Sandeep K. Gupta, Shubhlakshmi, "A New Gabor Wavelet Transform Feature Extraction Technique for Ear Biometrics Recognition", 7th IEEE International Conference on Power India International Conference, 2014

[6] ] Dosodia, Priya, Amarjeet Poonia, Sandeep K. Gupta, and Shubh Lakshmi Agrwal. "New Gabor-DCT feature extraction technique for facial expression recognition." In Communication Systems and Network Technologies (CSNT), 2015 Fifth International Conference on, pp. 546-549. IEEE, 2015.

[7] D Verma, V.P. Dhaka, S. Agrwal, "An Improved average Gabor Wavelet filter Feature Extraction Technique for Facial Expression Recognition", International Journal on Innovations in Engineering and Technology, Volume-2 issue-4,2013 (ISSN: 2319-1058).

[8] ShubhLakshmi Agrwal, Anita Yadav, Umesh Kumar, Sandeep Kumar Gupta,“ Improved Invisible Watermarking Technique Using IWT-DCT”, 5th International Conference on Reliability, Infocom Technologies and Optimization(ICRITO), IEEE, 2016.

[9] S. L. Agrwal, M. Sharma, D. Kumari and S. K. Gupta, "Improved image compression technique using IWTDCT transformation," 2016 2nd International Conference on Next Generation Computing Technologies (NGCT), Dehradun, India, 2016, pp. 683686.

[10] N. Janu, S.K. Gupta, P. mathur, S.L. Agrwal , Performance Analysis of Frequency Domain based Feature Extraction Techniques for Facial Expression Recognition", International Conference on CONFLUENCE, IEEE, 2017.

[11] Sandeep, Shubhlakshmi, Yogesh, Neeta, "A Hybrid method of Feature Extraction for Facial Expression Recognition", in seventh international conference on Signal image Technology and Internet based systems (SITIS), page(s): 422 - 425, IEEE, 2011.

[12] Arbab-Zavar, Banafshe, and Mark S. Nixon. "Robust log-gabor filter for ear biometrics." In Pattern Recognition, 2008. ICPR 2008. 19th International Conference on, pp. 1-4. IEEE, 2008.

[13] Kumar, Ajay, and Chenye Wu. "Automated human identification using ear imaging." Pattern Recognition 45, no. 3 (2012): 956-968.

[14] Preksha Tambi, Savita shiwani, Sandeep G.," Performance Analysis of K-means Clustering and Kmeans clustering with Improved Initial means", International Journal of Engineering Research and Technology, Vol.- 2, Issue-4, Page no-128-131, ISRSA 2013, (ISSN:2278-0181). 